laboratory investigations. Conversely, aortic dissection is not often considered a cause of myocardial malperfusion; thus misdiagnosis, which represents one of the main preoperative risks for these patients, may frequently occur. ${ }^{1}$ This was the case for our patient, to whom systemic thrombolytic treatment was given before a diagnosis of acute aortic dissection was made.

The subsequent surgical treatment was strongly influenced by this inadvertent treatment, which induced us to avoid any prosthetic material to replace the ascending aorta and imposed a closed technique of anastomosis to avoid hypothermic circulatory arrest. It should be said, however, that the limited longitudinal extension of the dissection was well suited to the repair technique.

This procedure does not fulfill the radical criteria advocated by some authors in the settings of acute type A dissection. ${ }^{6}$ However, it should be underlined that the resection included the entire dissected portion of the aorta and that the parts left in place exhibited a completely normal aspect.

\section{References}

1. Neri E, Toscano T, Papalia U, Frati G, Massetti M, Capannini G, et al. Proximal aortic dissection with coronary malperfusion: presentation, management, and outcome. J Thorac Cardiovasc Surg. 2001;121:55260.

2. Rizzo RJ, Aranki SF, Aklog L, Couper GS, Adams DH, Collins JJ Jr, et al. Rapid noninvasive diagnosis and surgical repair of acute ascending aortic dissection: improved survival with less angiography. J Thorac Cardiovasc Surg. 1994;108:567-75.

3. Borst HG. Organ ischemie. In: Borst HG, Heinemann MK, Stone CD, editors. Surgical treatment of aortic dissection. 1st ed. New York: Churchill Livingstone; 1996. p. 249-64.

4. Hufnagel CA, Conrad PW. Intimo-intimal intussusception in dissecting aneurysm. Am J Surg. 1962;103:723-7.

5. Neri E, Capannini G, Carone E, Tucci E, Diciolla F, Sassi C. The missing flap: considerations about a case of aortic intussusception. J Thorac Cardiovasc Surg. 1999;117:829-30.

6. Westaby S, Saito S, Katsumata T. Acute type A dissection: conservative methods provide consistently low mortality. Ann Thorac Surg. 2002; 73:707-13.

\title{
The frozen elephant trunk technique: A new treatment for thoracic aortic aneurysms
}

\author{
Matthias Karck, MD, ${ }^{\text {a }}$ Ajay Chavan, MD, ${ }^{\text {b }}$ Christian Hagl, MD, ${ }^{a}$ Holger Friedrich, MD, ${ }^{a}$ Michael Galanski, MD, ${ }^{\mathrm{b}}$ and \\ Axel Haverich, MD, ${ }^{a}$ Hannover, Germany
}

ombined diseases of the aortic arch and the descending aorta remain among the great challenges facing cardiothoracic surgeons. In 1983, Borst and associates ${ }^{1}$ introduced the two-stage elephant trunk principle into
cardiovascular surgery, which has greatly facilitated surgical treatment of this special pathologic entity. This approach is based on the prosthetic replacement of the ascending aorta and aortic arch with an elephant trunk extension of the arch graft inserted into the descending aorta during the first-stage operation performed through a median sternotomy. However, the graft segment forming the elephant trunk is floating freely in the descending aortic lumen, thus impeding thrombus formation between the graft and the aneurysmal vessel wall. Because thrombotic occlusion of this space is a prerequisite for reduction of wall stress of the aneurysmal vessel-thereby preventing subsequent growth of the aortic

\footnotetext{
From the Divisions of Thoracic and Cardiovascular Surgery a and Radiology, ${ }^{\mathrm{b}}$ Hannover Medical School, Hannover, Germany.

Received for publication June 26, 2002; accepted for publication July 7, 2002.

Address for reprints: Matthias Karck, MD, Division of Thoracic and Cardiovascular Surgery, Hannover Medical School, Carl-Neuberg-Strasse 1, Hannover 30625, Germany (E-mail: Karck@thg.mh-hannover.de).

J Thorac Cardiovasc Surg 2003;125:1550-3

Copyright $\odot 2003$ by The American Association for Thoracic Surgery $0022-5223 / 2003 \$ 30.00+0$

doi:10.1016/S0022-5223(03)00045-X
}

diameter ${ }^{2}$ - the elephant trunk prosthesis either has to be connected directly to the distal descending portion of the aorta or needs to be extended to the desired level requiring the secondstage operation through a left thoracotomy.

It is evident that comorbidity is a relevant issue in patients with aneurysmal diseases of the aorta, so new therapies to reduce the surgical trauma and risk are desirable. We describe a new approach that allows complete surgical treatment of such combined lesions during a single operation (through a median sternotomy) by using a prototype stent graft that can be placed into the descending aorta in an antegrade fashion through the opened aortic arch.

\section{Methods}

Between September and November 2001, a total of 4 patients were operated on after approval was obtained from the institutional review board of Hannover Medical School. Informed consent was obtained in each case.

In each case spiral computed tomographic angiography of the thoracic aorta was performed before the operation to demonstrate the extent of aortic pathologic process and to determine the correct stent graft size. A custom-made, prototype Chavan-Haverich $(\mathrm{CH})$ stent graft was used for the procedure. The $\mathrm{CH}$ stent graft (Curative Medical Devices Gmbh, Dresden, Germany) consisted of a Dacron polyester fabric vascular prosthesis with stainless steel stents affixed on the inner aspect at its distal end with the help of polypropylene sutures. All stents were fully covered with the Dacron prosthesis. The proximal portion of the $\mathrm{CH}$ stent graft was composed merely of a Dacron tube. The length of the stent graft 
was at least $5 \mathrm{~cm}$ greater than the estimated length of the aortic segment to be bridged. The redundant proximal portion of the Dacron tube was trimmed to an appropriate length and surgically sutured to the aortic wall or to a proximal aortic vascular prosthesis. The total lengths of the stent grafts varied between 200 and 286 $\mathrm{mm}$. The diameter of the proximal end of the $\mathrm{CH}$ stent graft was chosen to match the diameter of the proximal aorta or of the proximal surgical graft in the aortic arch to which the $\mathrm{CH}$ stent graft had to be sutured. These diameters were $36 \mathrm{~mm}$ in 3 cases and $30 \mathrm{~mm}$ in 1 case.

The distal stents had a length of $22 \mathrm{~mm}$ each; a gap of $5 \mathrm{~mm}$ between adjacent stents provided flexibility of the system. The distance between the stents was maintained with the help of two longitudinally oriented stainless steel wires. Depending on individual patient anatomy, the number of stents varied between three and four. To determine the diameters of the distal stents, the diameter of the descending aorta at the site of intended distal docking was used. The diameters of the distal stents were $36 \mathrm{~mm}$ in 2 cases and 44 and $46 \mathrm{~mm}$ in 1 case each.

The delivery system for the $\mathrm{CH}$ stent graft was flexible and could be advanced into the descending aorta over a 0.035 -inch super-stiff guide wire (Back-up Meier; Boston Scientific Corporation, Boston, Mass), and possessed a "sheath in sheath" design. It consisted of an outer $30 \mathrm{~F}$ sheath, an inner $25 \mathrm{~F}$ sheath, and a central pusher. Withdrawal of the 30F sheath while the inner sheath and the pusher were held steady released the stented portion of the $\mathrm{CH}$ stent graft. The proximal Dacron tube could then be released by pulling back both the sheaths simultaneously while holding the pusher steady. After deployment, the stented portion of the $\mathrm{CH}$ endograft was modulated onto the aortic wall with the help of an appropriately sized balloon catheter (Medtronic, Inc, Minneapolis, Minn). The individual surgical procedures are described in detail in the clinical summaries.

\section{Clinical Summaries}

PATIENT 1. Patient 1 was a 71-year-old woman with an aneurysm of the descending aorta (maximum diameter $6 \mathrm{~cm}$ ) starting at the origin of the left subclavian artery, combined with coronary artery disease. She underwent open antegrade stent graft implantation into the descending aorta and double coronary artery bypass grafting. After median sternotomy, extracorporeal circulation (ECC) was established after cannulation of the ascending aorta and the right atrium. During core cooling to $20^{\circ} \mathrm{C}$, double coronary artery bypass grafting was performed. After the target core temperature was reached, ECC was discontinued and selective antegrade cold blood brain perfusion was initiated. Then the stent graft was delivered through the opened aortic arch, and its distal end was anchored at the level of the 10th thoracic vertebra under radiographic control. The proximal, nonstented Dacron graft segment was sutured circumferentially directly distal to the origin of the left subclavian artery; the aortotomy was then closed, and the operation was continued in the usual fashion.

PATIENT 2. Patient 2 was a 50-year-old man with chronic type A aortic dissection. He had critical aortic dilatation proximal to a previously implanted ascending aortic prosthesis as well as the aortic arch and the proximal descending aorta. After resternotomy, ECC was established after cannulation of the aortic arch and the right atrium. After the target temperature of $24^{\circ} \mathrm{C}$ was reached,
ECC was discontinued. After administration of cold blood cardioplegia, opening of the aortic arch to the level of the left subclavian artery, and institution of selective antegrade cold blood brain perfusion, the stent graft was delivered into the true lumen to the level of the 9th vertebra under radiographic control. The proximal nonstented part of the prosthesis was sutured directly into the aortic orifice distal to the left subclavian artery. Then a second precoated graft was implanted to replace the aortic arch and the previously implanted ascending aortic prosthesis.

PATIEnTs 3 And 4. Patients 3 (Figure 1) and 4 were 63- and 65 -year-old women with aneurysms of the ascending aorta, the aortic arch, and the proximal descending aorta. In addition, patient 3 had single-vessel coronary artery disease. ECC was established by cannulation of the aortic arch and the right atrium. After core cooling to $26^{\circ} \mathrm{C}$, ECC was discontinued. After administration of blood cardioplegia, opening of the arch to the level of the left subclavian artery, and institution of selective antegrade cold blood brain perfusion, the stent graft was delivered into the descending aorta down to level of the 8th thoracic vertebra under radiographic control, with the proximal, nonstented part of the prosthesis subsequently sutured inside the aorta directly distal to the left subclavian artery. Then a second precoated nonstented vascular graft was implanted to replace both the aortic arch and ascending aorta. After cannulation of the prosthesis and reconstitution of ECC, a single venous graft to the left anterior descending coronary artery was implanted in patient 3 .

\section{Results}

There were no intraoperative or postoperative deaths. Mean cardiopulmonary bypass time was $234 \pm 58$ minutes, aortic crossclamp time was $130 \pm 41$ minutes, circulatory arrest time was 87 \pm 39 minutes, and time of selective antegrade brain perfusion during circulatory arrest was $69 \pm 43$ minutes. Patients 1,2 , and 4 were weaned from the respirator within 48 hours after the operation. Their further courses were uneventful, with no neurologic complications. Patient 3 had a temporary postoperative neurologic dysfunction, as indicated by prolonged intubation followed by moderate confusion and agitation. Two postoperative cerebral computed tomographic scans yielded negative results, and clinical symptoms had resolved completely by discharge on postoperative day 29.

In the 3 patients with aneurysms of the descending aorta, the follow-up computed tomographic scans revealed complete exclusion of the aneurysms. In the patient with the aortic dissection, initiation of thrombus formation in the false lumen in the cranial sections of the descending aorta was noted at 3 months of followup. Below the level of the stents, however, persistent perfusion of the false lumen was noted. The diameter of the descending aorta did not increase at the end of follow-up (2-5 months).

\section{Discussion}

Thoracic aortic aneurysms are still a challenge for the surgeon, particularly when critical dilatation of the ascending aorta and arch extends beyond the level of the left subclavian artery. Treatment either requires a two-stage approach with the elephant trunk principle or an extensive and traumatic single-stage procedure, generally performed through a clamshell incision. ${ }^{3-5}$ 


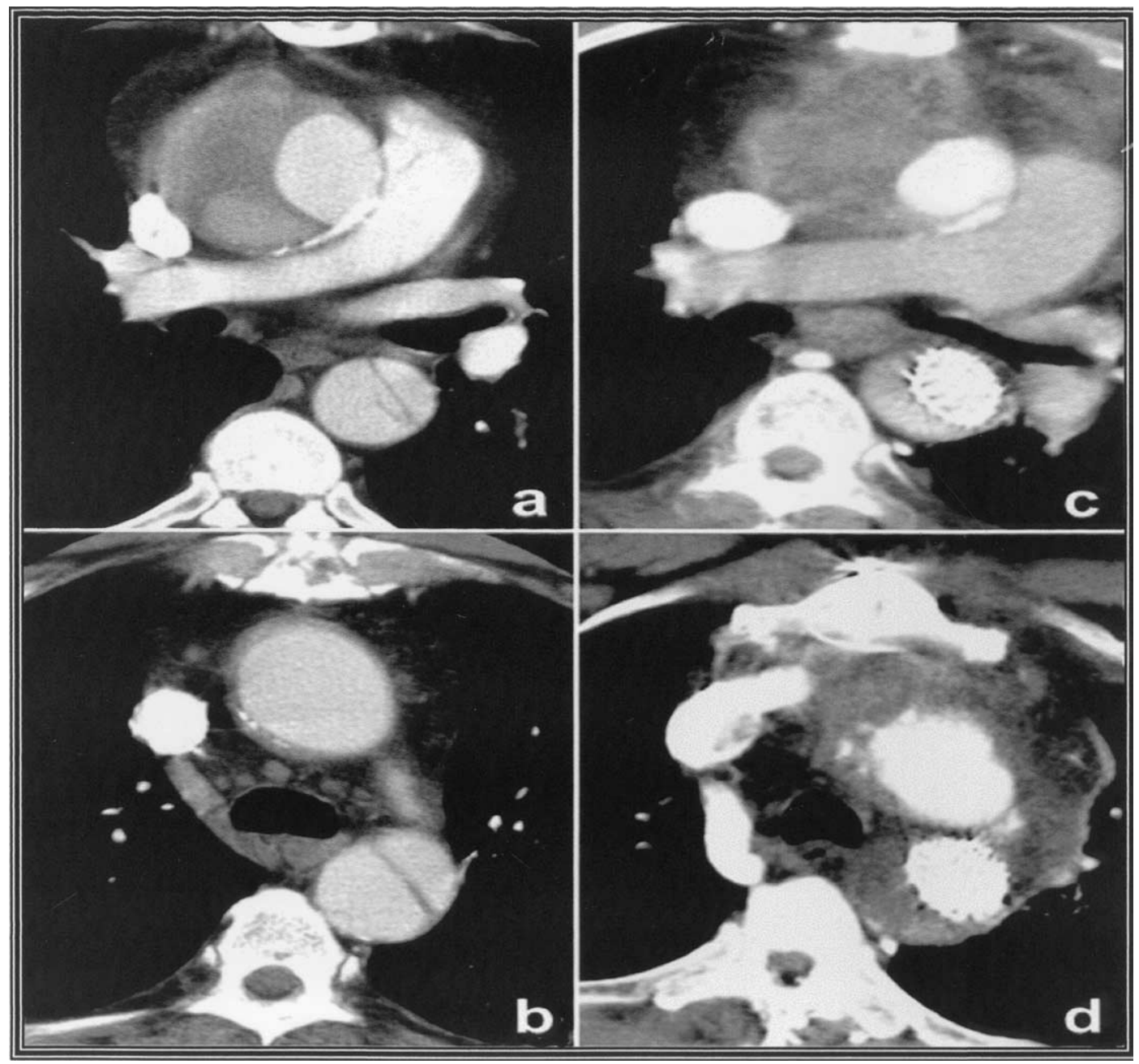

Figure 1. Axial computed tomographic sections of patient 3 with aneurysms of ascending aorta (a) as well as of proximal descending aorta commencing at origin of left subclavian artery (b). After open surgical replacement of ascending aorta (c) and placement of $\mathrm{CH}$ stent graft in aortic arch and descending aorta (c), note that aneurysm of descending aorta has thrombosed completely around $\mathrm{CH}$ graft (d).

Here we have described our early experience with a new approach that allows definitive treatment of such combined lesions during a single-stage procedure that includes antegrade placement of a stented graft into the descending aorta through the opened aortic arch during hypothermic circulatory arrest. This procedure can be performed through a conventional median sternotomy and combines the concepts of the elephant trunk principle and endovascular stenting of descending aortic aneurysms. ${ }^{1,6}$ Its advantage lies in the fact that the stented graft, unlike a conventional elephant trunk prosthesis, can be securely anchored at the desired level distal to the descending aortic aneurysm, thereby allowing thrombus formation within the space between the graft and the wall of the aneurysm. The inventor of the elephant trunk technique, Hans G. Borst, has named this approach the "frozen elephant trunk" (unpublished data). The proximal, nonstented portion of the graft is sutured circumferentially into the aorta directly distal to the left subclavian artery with a similar technique to that suggested by
Palma for treatment of acute type B aortic dissection. ${ }^{7}$ Orihashi and colleagues ${ }^{8}$ have also used this approach, under debatable circumstances, however, considering the relatively small and circumscribed aneurysms being excluded. Unlike in their study, most of our patients had to undergo simultaneous replacement of the aneurysmal upstream aorta by implantation of a second vascular prosthesis. This procedure allows single-stage treatment of extensive thoracic aneurysms that otherwise would require either a two-stage procedure or a much more complex single-stage operation.

Except for a fully reversible temporary neurologic dysfunction in patient 3 , there was no postoperative morbidity that could be attributed to the fact that stent graft placement currently requires an additional 20 minutes of circulatory arrest. However, we believe that antegrade selective cold blood brain perfusion ${ }^{9}$ is an essential tool to protect the brain from ischemic neuronal cell damage. $^{10,11}$ 
Encouraged by the favorable results of this limited series, we evaluate all patients with thoracic aortic aneurysms extending proximal and distal of the left subclavian artery for this new treatment modality, the frozen elephant trunk technique.

\section{References}

1. Borst HG, Walterbusch G, Schaps D. Extensive aortic replacement using the "elephant trunk" prosthesis. Thorac Cardiovasc Surg. 1983; 31:37-40.

2. Parodi JC, Palmaz JC, Barone HD. Transfemoral intraluminal graft implantation for abdominal aortic aneurysms. Ann Vasc Surg. 1991; 5:491-9.

3. Coselli JS, Oberwalder P. Successful repair of mega aorta using reversed elephant trunk procedure. J Vasc Surg. 1998;27:183-8.

4. Westaby S, Katsumata T. Proximal aortic perfusion for complex arch and descending aortic disease. J Thorac Cardiovasc Surg. 1998;115:162-7.

5. Minale C, Splittberger F, Wendt G, Messmer BJ. One-stage intrathoracic repair of extended aortic aneurysms. J Card Surg. 1994;9:604-13.
6. Dake MD, Miller DC, Semba CP, Mitchell RS, Walker PJ, Lindell HP. Transluminal placement of endovascular stent-grafts for the treatment of descending thoracic aortic aneurysms. N Engl J Med. 1994;331: 1729-34.

7. Palma JH, Almeida DR, Carvalho AC, Andrade JC, Buffolo E. Surgical treatment of acute type B aortic dissection using an endoprosthesis (elephant trunk). Ann Thorac Surg. 1997;63:1081-4.

8. Orihashi K, Sueda T, Watari M, Okada K, Ishii O, Matsuura Y. Endovascular stent-grafting via the aortic arch for distal aortic arch aneurysm: an alternative to endovascular stent-grafting. Eur J Cardiothorac Surg. 2001;20:973-8.

9. Griepp RB. Cerebral protection during aortic arch surgery. $J$ Thorac Cardiovasc Surg. 2001;121:425-7.

10. Bachet J, Guilmet D, Goudot B, Dreyfus GD, Delentdecker P, Brodaty $\mathrm{D}$, et al. Antegrade cerebral perfusion with cold blood: a 13-year experience. Ann Thorac Surg. 1999;67:1874-8.

11. Hagl C, Ergin MA, Galla JD, Lansman SL, McCullough JN, Spielvogel $\mathrm{D}$, et al. Neurologic outcome after ascending aorta-aortic arch operations: effect of brain protection technique in high-risk patients. J Thorac Cardiovasc Surg. 2001;121:1107-21.

\title{
Mitral valve-sparing operation in subaortic stenosis caused by anomalous papillary muscle and discrete subaortic stenosis
}

\author{
Jen-Ping Chang, MD, Hung-I Lu, MD, Chiung-Lun Kao, MD, and Teng-Hung Yu, MD, Taiwan, Republic of China
}

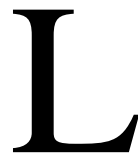

eft ventricular outflow tract (LVOT) obstruction caused by subaortic stenosis covers a wide range of anatomic lesions. Most cases in children were caused by the discrete subaortic stenosis (DSAS). ${ }^{1}$ In adult patients LVOT obstruction caused by anomalous insertion of papillary muscle into the base of the anterior mitral leaflet in hypertrophic cardiomyopathy has been reported. ${ }^{2,3}$ Isolated LVOT obstruction caused by an anomalous papillary muscle (APM) has been recognized also. ${ }^{4,5}$ The procedures, including entire resection of the APM and mitral valve replacement, have been thought necessary for the successful correction. Report of LVOT obstruction caused by the combination of DSAS and APM is rare. ${ }^{6}$ We report on such a patient who was treated with extensive resection of the APM, resection of the DSAS, and careful mobilization of the anterior mitral leaflet, resulting in successful relief of the obstruction without mitral valve replacement.

From the Division of Thoracic and Cardiovascular Surgery, Cardiology, Chang Gung Memorial Hospital at Kaohsiung, Taiwan, Republic of China.

Received for publication Sept 17, 2002; accepted for publication Oct 3, 2002.

Address for reprints: Jen-Ping Chang, MD, Division of Thoracic and Cardiovascular Surgery, Chang Gung Memorial Hospital at Kaohsiung, 123, Ta-Pei Rd, Niao Sung Hsiang, Kaohsiung Hsien, Taiwan, Republic of China (E-mail: c9112772@adm.cgmh.org.tw)

J Thorac Cardiovasc Surg 2003;125:1553-5

Copyright $\odot 2003$ by The American Association for Thoracic Surgery $0022-5223 / 2003 \$ 30.00+0$

doi:10.1016/S0022-5223(03)00010-2

\section{Clinical Summary}

A 48-year-old woman presented with progressive shortness of breath on exertion and chest tightness over a period of 3 years. Holter monitoring showed sinus rhythm. A 12-lead electrocardiogram revealed voltage criteria for left ventricular hypertrophy with strain pattern. On auscultation, a grade $4 / 6$ harsh systolic murmur maximal at the right upper sternal border was audible. Transthoracic echocardiography showed that the LVOT was obstructed by the membranous DSAS and an anomalous insertion of a papillary muscle to the anterior mitral leaflet with a peak instantaneous gradient of $90 \mathrm{~mm} \mathrm{Hg}$ (Figure 1). The aortic valve had 3 cusps and was normal in size and appearance. The ventricular septum and the left ventricular posterior wall were mildly thickened. Neither systolic anterior motion nor localized hypertrophy of the ventricular septum was noted. Coronary angiographic results were normal.

The operation was performed through a lower half ministernotomy with standard cardiopulmonary bypass and cold oxygenated crystalloid cardioplegic arrest. On inspection through the oblique aortotomy, the aortic valve was found to be mildly sclerotic and competent. The LVOT was explored, and a papillary muscle 12 $\mathrm{mm}$ in diameter was found extending from the anterior mitral leaflet to the apicoseptal portion of the left ventricle without any chordal interposition. A membranous DSAS $6 \mathrm{~mm}$ in width was found just below the left coronary cusp as well (Figure 2). The APM and the DSAS were resected. Then an extensive myectomy trough was carried within the left ventricular midcavity. Finally, the anterior mitral leaflet was fully mobilized, with careful shaving off of the residual papillary muscle and the secondary chords, which were attached over the ventricular surface of the leaflet (Figure 3). Cardiopulmonary bypass was discontinued unevent- 